\section{The Eurth's Cores}

THE inner core of the Earth has for too long lived in the shadow of the outer core. A great deal is known about the outer core: it is liquid, it has a radius of $3,480 \mathrm{~km}$ and it is almost certainly made mostly of molten iron. The inner core was detected because some seismic energy was recorded by seismographs lying between $105^{\circ}$ and $143^{\circ}$ from earthquakes. In the absence of an inner core these instruments should have lain in the shadow of the core and have received no energy. It seemed impossible that the energy was diffracted into the shadow and it was postulated that there was an inner core with a radius of $1,200 \mathrm{~km}$, from the surface of which the seismic waves were reflected.

Having found or suspected that the Earth has an inner core it was natural to enquire whether it is solid or liquid. There was a certain convenience in having it solid, because it could then be supposed to be made of the same material as the outer core and to be solidified by pressure. This was an easier view to explain to undergraduates than the alternative that the two cores were composed of two immiscible liquids. Are there immiscible liquids at high pressures and temperatures? Maybe not, but who can tell? It is possible that a liquid can be formed from molten iron by collapsing the partially filled D-shell of extranuclear electrons; maybe it will not mix with ordinary molten iron. It would seem that the matter could easily be settled by showing that both compressional and distortional waves can travel through the inner core ; to transmit distortional waves is an essential property of a solid. The distortional waves have not in fact been found, but the seismogram fanciers are not willing to say that they are absent. In these circumstances, those people not immediately concerned mostly concluded that very little was known about the inner core and that it was best left to the experts till its properties became clearer.

In this issue of Nature (page 465) Dziewonski and Gilbert provide strong evidence for the solidity of the inner core. The Earth possesses "normal modes" which are excited by earthquakes. To deduce the structure of the Earth from them is rather like deducing the structure of a piano from the noise it makes when thrown downstairs. It is possible because there are already rather good models of the Earth derived from seismic travel times. Dziewonski and Gilbert have picked out particular kinds of vibrations which have an appreciable part of their energy (up to 20 per cent) in the inner core and the frequency of which therefore depends markedly on its properties. The results are entirely compatible with a solid inner core and Dziewonski and Gilbert believe that no model with a liquid inner core can satisfy all the data.

Dziewonski and Gilbert's model is important. If the transition from the inner to the outer core is a transition from the solid to the liquid forms of a single material, then the boundary must be at the melting point and a constraint is put on the thermodynamics of the Earth's interior. That the boundary is at the melting point is an essential assumption in an argument put forward by Higgins and Kennedy (J. Geophys. Res., 76, $1870 ; 1971)$ which suggests that the outer core is stably stratified. If this is so the large-scale steady motions usually postulated as constituting the dynamo that produces the Earth's magnetic field are presumably not possible (for a possible escape from this misfortune, see Bullard and Gubbins, Nature, 232, 548 ; 1971).

The very existence of an iron core suggests ideas on the early history of the Earth. It is unlikely that the Earth accumulated in two stages, first particles of iron forming the core and then particles of silicate forming the mantle. Whatever the exact mechanism it seems likely that at the start the particles of iron and silicate were mixed up. If the accumulation were very rapid or if it took place in a very opaque atmosphere, the energy of impact would cause the particles to melt and could lead to separation of the core beneath the lighter mantle just as iron separates from slag in a blast furnace. Alternatively, if the rate of accumulation is slow the gravitational energy of each incoming particle will be radiated away and the Earth will accumulate as a solid with a temperature of a few hundred degrees. It is then possible that radioactivity will cause melting and separation of the core after a time of the order of $10^{8}$ years. There are therefore two possible views about the history of the core ; it could have formed as the Earth accumulated or its formation could have been a subsequent event. The second is the fashionable view, though it cannot be said that the arguments in its favour are conclusive.

On page 463 of this issue, Oversby and Ringwood argue for the first view - the very early formation of the core. They point out that the formation of the core would remove lead from the mantle and decrease the $\mathrm{Pb} / \mathrm{U}$ ratio. The lead at present in the mantle is partly that left after the formation of the core and partly that produced by decay of uranium and thorium in the mantle. If the original isotopic composition of the lead incorporated in the Earth, the present composition of the lead in the mantle and the proportion of lead removed in forming the core are known, then, according to Oversby and Ringwood, the time of formation of the core can be found. These quantities can, with varying degrees of plausibility, be said to be known, and the result favours an early formation of the core.

Details of the argument will be awaited with interest. The formation of the core while the outer part of the Earth was solid would have been a cataclysmic event (there is a large release of energy); it must have occurred before any of the rocks now at the Earth's surface were formed. The ages of the oldest known rocks have been increasing and are now about 3,980 million years. The margin between this figure and the age of the Earth (4,550 million years) is becoming uncomfortably narrow and early core formation seems, on these grounds, desirable. 